\title{
1. Introduction: framing resilience research
}

Edward H. Powley

Resilience has grown in popularity, interest and application in a wide variety of scholarly and practitioner domains. This Research Handbook samples resilience scholars who are conceptualizing and exploring various ways to enliven and embed resilience in theory and practice. Contributors to this volume represent an international group of scholars who offer new and updated perspectives. Some propose new contexts for resilience, while others combine theoretical frames to bring new insights to the field, and still others offer perspectives on the practice of resilience.

Definitions of resilience vary depending on the phenomenon or context under study. Britt and Sawhney note (Chapter 2) that the 'increase in interest has been accompanied by multiple definitions of resilience, as well as confusion regarding whether resilience is best viewed as an attribute of employees, a process that occurs when employees are exposed to high levels of adversity at work, or an outcome that is demonstrated following such adversity.' For some in this volume, resilience is about individual characteristics, traits or states, such as grit, emotional regulation, responding to stress, adapting under pressure or adversity, or learning from setbacks and challenges. For others, resilience is relationally derived and comes about through social interaction and the ebb and flow of group, team or organizational dynamics. Resilience theory at the team or group level is nascent and thus offers conceptual ways to address theory development. And still yet for other contributors, resilience is an attribute or defining characteristic of an organization or system capacity to adapt and adjust to the environment and take and integrate feedback necessary to maintain a positive trajectory. Moreover, resilience describes a response to adverse situations, such as major disruption and crisis (earthquakes, disasters, technological). And yet, resilience equally evokes a proactive capacity or cultural dynamic that promotes and fosters learning, adaptation, or flexibility to build teams and organizations that are likely to face future challenges. Most importantly, resilience cannot be defined without the presence of some kind of stressor, whatever that might be.

What we notice is that while resilience is varied and nuanced given context and framing, despite differences in definitions there are several shared features of resilience: resilience is about positive adjustment under crisis or stress, and bouncing back to a new stable state. Regardless of the domain or level of analysis, resilience serves as a maintenance function to ensure a stable state (Maitlis, 2012; Westphal and Bonanno, 2007; Guarnieri and Travedel, 2018). Resilience involves learning, growth, efficacy and competence (Sutcliffe and Vogus, 2003; Janoff-Bulman, 1985, 1992; Tugade and Fredrickson, 2004) and the ability to bounce back (Gittell et al., 2006; Zolli and Healy, 2012; Powley, 2009). Resilience scholars, regardless of discipline, return to these conceptual roots, while the operationalization and mechanisms by which resilience unfolds, manifests itself or operates, differ depending on where and how one studies resilience. The core questions for scholars remain: when we study resilience, what are we studying? Is it the person, the group, the system, a dynamic, a relationship, a fixed trait, a process, a state? Is resilience as a concept capable of describing such a wide variety of contexts and issues, or might there be other concepts better suited to describe the phenom- 
enon? These persistent questions drive our theorizing and eventual practice in this field, and the contributors to this Research Handbook wrestle with these fundamental issues, offering valuable perspectives on resilience while addressing implications for practice and research.

We have purposively selected contributors who examine resilience in historically traditional ways: at the individual, group and team, and organizational levels of analysis; but we have also included chapters that explore resilience in inter-organizational systems, major crisis situations, and cultural dynamics where collaboration and strategic relationships are important. Our primary framing situates resilience in the organizational sciences, that is, our work concerns resilience in and of organizations such that the chapters offer conceptual insights and research findings about resilience in an organizational context. Each chapter frames resilience in ways that are consistent with these overarching themes, yet each offers new understandings and contexts for resilience. Included are conceptual frameworks, case studies, and qualitative and quantitative studies that span from the individual level of analysis to macro and inter-organizational systems. The chapters are organized into three broad categories and each of the chapters is summarized in the next three sections of the introduction. The second part of the book addresses the nature of resilience broadly where authors offer important considerations on the definition and framing of resilience. The third part includes chapters framing resilience as process and explores dynamics of resilience. The fourth part focuses on antecedents and outcomes of resilience. A concluding chapter offers a roadmap for future research and poses several questions scholars might consider to advance resilience research.

\section{PART II: NATURE OF RESILIENCE}

The second part of the Research Handbook is concerned with the nature of resilience. Chapters highlight definitions of resilience in various contexts to help clarify the meaning and manifestations of resilience. Authors address such questions as: Does the meaning and content of resilience differ or is it stable given the context or level of analysis? Is resilience a construct that is uniquely individual? Or is resilience relationally generated through interpersonal interactions and social dynamics? Some chapters consider resilience as more than persisting through adverse situations but rather see positive potential for change and adjustment.

Britt and Sawhney provide a roadmap to integrate resilience across levels of analysis. A key for resilience is the presence of adversity, setback or disruption, regardless of where or at what level one examines resilience. The chapter looks across levels of analysis from individual, to team, to the organization to map the study of resilience in and of organizations. Moreover, the authors distinguish between the demonstration, capacity and processes responsible for resilience: how it is shown, how it is stored, and how it unfolds. The chapter outlines a multi-level model of resilience and calls for more research to further clarify resilience scholarship.

Caza, Caza and Baloochi focus on grit as one aspect of the nature of a resilient personality, and report on an empirical study to show the relationship between grit and resilience. Grit, defined as passion and perseverance toward the achievement of long-range goals, is closely associated with resilience, and the research study differentiates grit from hardiness, a well-established measure of trait resilience, and also, by understanding its relationship to individual outcomes indicative of resilient responses. Results show that grit is not an indicator of resilient outcomes, and that grit and resilience may be two different but related constructs. 


\section{Research handbook on organizational resilience}

Hartmann, Weiss and Hoegl present a conceptual model of team resilience that highlights the collective dynamics inherent in team interactions. Rather than an individual trait characteristic, the authors suggest that resilience is relationally derived, developing through team members' interactions. Taking a team process perspective, the authors develop an integrative conceptual framework and highlight how team resilience develops and changes through affective, social and cognitive team processes, and how team resilience may change over time.

Frigotto advances a framework of resilience from the perspective of novelty and change, where 'novelty is used to elaborate on the type and nature of adversities that trigger resilience, and change is used to elaborate on the type and nature of the resilience outcomes.' Resilience is often associated with bouncing back and maintaining equilibrium, which implies returning to a former state of normal operations, but resilience may result in three different change outcomes: absorptive, adaptive and transformative. The chapter also depicts resilience as emergent and evolving and characterizes it as a renewable and ever-changing resource.

Neville, Caza and Olekalns discuss resilience in the context of negotiation adversity and its negative consequences. The chapter posits that negotiation resilience is a cognitive process of the individual negotiator. Key to their conceptualization of resilience of an individual actor is the notion of persistence and then shifting or adjusting in the face of adversity. Adversity is viewed as a positive interruption that prompts flexibility and adjustment. What is generated is positive growth allowing negotiators to move forward, perhaps with greater ease and confidence. The chapter identifies key predictors of negotiation resilience: growth narrative, cost-benefit analysis, and flexible thinking, and offers suggestions about the development of resilience-building interventions for negotiators.

\section{PART III: RESILIENCE PROCESSES AND DYNAMICS}

More than an individual trait, state, attribute or characteristic, resilience processes and dynamics are another way to view resilience conceptually. Resilience dynamics are not unique to the individual or interpersonal relationships, but are also played out across organizational boundaries. Chapters in the third part of the Research Handbook describe how resilience is reflected in processes with mediating and moderating factors in various contexts. Authors offer insights on resilience dynamics in unique and new contexts - places where resilience matters for the well-being and livelihoods of individuals, their workplaces, and for organizations broadly. As the chapters show, resilience dynamics are an important aspect of emotion regulation and using tacit knowledge from sensory information to manage adversity. Moreover, resilience dynamics are as important in new businesses in emerging economies, community organizations facing natural disasters, or long-term stakeholder relationships, as they are in vulnerable populations who face structural obstacles or contexts of diversity crisis.

Kay and Merlo examine emotion regulation as a process to enable resilience. Challenges, setbacks, or other significant disruptions have positive or negative emotional implications. Managing negative emotions, or emotion regulation, is critical to manifesting resilience. The chapter draws on the process of emotion regulation, including emotion episodes and regulation strategies to explain the role of emotional experience in resilience. The authors propose ways in which individuals and organizations might foster emotion regulation strategies to foster resilience. 
Stephens offers a process theory for how individuals use sensory information and embodied expressions to make sense of situations at work. The chapter argues that tacit knowledge and sensory information advances knowledge of cross-level dynamics that potentially affect organizational resilience. The chapter draws on the concept of relational pauses wherein group members reflect on and attend to others' sensory templates. As they do so, they enact adaptive moves based on the tacit knowledge embedded in their sensory templates. Resilience takes the form of process wherein adaptive moves may be shared with other individuals or groups that in turn directly impact the organization.

Becker and Kabongo provide an interesting view of resilience of entrepreneurs in emerging and developing economies - a rich context where the ability to manage insurmountable obstacles results in either decline and failure or survival and growth. New business leaders face competition, economic disruption, or logistics and supply challenges, not unlike larger corporations but at the local level. 'Entrepreneurial resilience' is a capacity of those who start businesses 'to demonstrate concrete, positive adaptation to adversity,' and is a highly sought characteristic in developing economies where additional contextual challenges, unstable political conditions, and local and specific challenges complicate business processes and the ability to bring a product to market. Using examples from developing economies, the chapter presents a model for resilience which identifies key factors necessary to foster resilience.

Hernandez, Baker, Hess and Harris describe a five-year longitudinal qualitative study of organizational resilience to explain how multi-stakeholder collaboration contributes to an organization's ability to bounce back from adversity. Managers draw on individual, organizational and stakeholder strategies to foster and develop resources. Embedded in the social and organizational context are stakeholder relationships based on shared affective connection which managers cultivate and then access and leverage in times of trial. Whereas resilience has often been viewed as a characteristic or an attribute of an organization, this chapter highlights a more nuanced view of resilience as an emergent dynamic in and between stakeholders whose social exchanges facilitate and support an organization's ability to work through challenges.

Linnenluecke and McKnight focus on resilience at the community level. Bridging resilience from different domains: business continuity, non-profit, and the public and private sectors, the chapter focuses on the role of firms in times of natural disaster. The authors draw from the literature on community resilience to conceptualize resilience as a process to anticipate, prepare for, react to, and recover from adversity. Several case studies highlight how organizational resilience is not solely situated within corporations or for-profit firms, but rather firm resilience has an important effect on other community organizations. The authors propose a framework to examine the interconnectedness between firms and community resilience and show that firms play a role in improving resilience at the community level in the context of disasters.

Johnson, Erskine and Roberts explore resilience in the context of vulnerable workers, in particular, Black women who often face structural obstacles arising from racism or sexism. The chapter looks at resilience through the lens of these vulnerable workers as they face different forms of adversity and trauma at work, in society, and in interpersonal relationships. Resilience is a core mechanism for women of color working though trauma and adversity. The authors also reflect on challenges that Black women face as they work to 'bounce back' from performance shortcomings and what accounts for their ability to overcome setbacks and failure, and they suggest potential interventions to improve the well-being of Black women in the workplace. 


\section{Research handbook on organizational resilience}

McCluney, Wooten and James offer an important perspective of resilience unfolding in the context of racial tensions in a university setting. The chapter brings into relief the resilience dynamics that came about during the recent diversity crisis at the University of Missouri (between 2014 and 2016). Using qualitative analysis, the authors show how resilience is a process which includes an ability to anticipate threats, cope effectively with unexpected events, and learn from these events. Analysis of public documents unveiled the lack of knowledge in higher education to address a diversity crisis, and how social media escalates crisis to a tipping point. The authors show how resilience creates dynamic capabilities and structures to facilitate organizational learning in preparation for future diversity-related crises.

\section{PART IV: RESILIENCE ANTECEDENTS AND OUTCOMES}

Resilience scholars conceptualize resilience as a key factor for producing positive results in challenging situations, or as the result or outcome when times are tough. Several chapters in this part view resilience as an outcome including factors such as learning, team processes, or individual differences. Other chapters focus on resilience as either an antecedent or independent variable, that is, factors that produce other positive behaviors or outcomes such as decreased stress, improved well-being, or citizenship behaviors. Whether an antecedent or outcome, scholars situate resilience research in the context of disruptive events or major crisis. Several of the chapters present case material from significant disaster or crisis contexts.

Kayes and Yoon outline learning routines that lead toward organizational resilience: openness to experience, learning identity, social support, flexibility, continuous improvement, and intentional focus. Moreover, resilience is embedded and woven through these learning routines such that as organizations engage in and develop learning routines, they are developing resilience. They argue that whereas organization resilience is often associated with bouncing back from breakdown, crisis or disruption, learning routines represent a positive pathway toward organizational resilience.

Jones Christensen, Hammond and Larsen draw comparisons between lost behavior in the context of wilderness and backcountry search and rescue, with lost behavior in the context of work organizations, and propose the concept of 'need for resilience.' When lost, individuals experience a range of emotions and engage in a variety of cognitive appraisals, with the express purpose of making sense of their 'lostness.' The chapter outlines how the need for resilience increases as individuals move deeper into psychological lostness, and that, as levels of lostness decrease, the need for resilience also decreases. Resilience acts as an antecedent whereby reunification and thriving after periods of lostness result from resilience increasing during periods of lostness.

Raetze suggests a model of team resilience wherein he articulates resilience processes and antecedents where adverse events may significantly disturb critical task- and team-related processes. The chapter gives an overview of research findings on work team resilience and proposes a conceptual multi-level framework. In particular, the chapter examines actions and capabilities that underlie the team resilience process: resilience is the outcome of team dynamics, processes, composition and design.

Karolidis, Vouzas and Antonacopoulou discuss resilience as an antecedent in the context of organizational citizenship behavior in public service employment. Public sector employment faces the unique challenge of having limited monetary and workforce resources, thus facing 
demands unlike other work organizations. The chapter describes individual resilience as the personal process of bouncing back, and argues that if resilience is coupled with organizational citizenship behaviors, public sector employees are more likely to be efficient and effective.

Powley and Cameron explore resilience in the context of organizational culture using the Competing Values Framework which provides one way to map competing conceptualizations of resilience. The competing values represent important factors to produce resilience culture types; each has value to organizations that face challenges and setbacks. Four key components of organizational resilience may be operative in each culture type to help activate resilience: leadership vision, organizational learning, mutual support, and ability to recover from setbacks. The chapter also highlights mechanisms that support a resilient organizational culture.

Three chapters present cases where resilience is an important factor in solving significant system challenges. Travadel and Guarnieri present an in-depth analysis of the actions and cognitions of Masao Yoshida, the plant superintendent of the Fukushima nuclear power plant, immediately following the earthquake and tsunami in March 2011. Their analysis outlines touchstone moments where Yoshida managed the delicate balance between what he did and did not know and critical decisions not only for plant safety but for much of eastern Japan. Using a phenomenological lens, Travadel and Guarnieri explore how Yoshida negotiated a disruptive threat, faced death, made sense of the impending danger and, ultimately, prevented a catastrophic situation from unfolding. The pressure and intensity of 'la situation extrême' placed Yoshida in a position where he was forced to give meaning to the flow of brute experience; such sense-in-the-making is the main driver of resilience.

Chesley and D'Avella describe a framework for large system resilience, what they refer to as resilience of inter-organizational systems. This chapter contributes to a broader conversation about resilience at levels of analysis beyond individuals, groups and organizations, and focuses on resilience of inter-organizational networks. To solve or address intractable problems such as climate, industrial accidents, or other natural or man-made crises, resilience at the macro level requires an integrative systems approach. The chapter - a mixed-method study of three inter-organizational systems working on sustainability and environmental projects draws out important considerations for managing critical relationships across organizational boundaries.

Walker, Malinen, Näswall, Nilakant and Kuntz examine 'resilience-in-action' from a multi-year, extended disaster recovery from a series of earthquakes near Canterbury, New Zealand. The chapter presents an in-depth qualitative survey that surfaces dynamics of resilience as they unfold, what they refer to as 'resilience-in-action,' resilience being the outcome of months of interventions and recovery efforts. Their grounded account of resilience-in-action outlines resilience trajectories of organizations and four areas that significantly influence organizational-level resilience: leadership and situational awareness; an employee-centric orientation; internal and external collaboration; and informal and organizational learning. The chapter also details a process model for the development of organizational resilience.

\section{CONCLUSION}

The final chapter poses key questions about conceptualizations of resilience. Resilience scholarship faces confusion: what do we mean by it, or how do we study it? The chapter discusses critical issues that aim to identify ways to study organizational resilience and resilience in 


\section{Research handbook on organizational resilience}

organizations. Due to challenges associated with defining or operationalizing resilience, the authors argue that researchers ought to pay more attention to challenges and key conceptual and empirical decisions when studying resilience. They suggest that scholars might concretely address key assumptions and operationalizations of resilience in terms of who, what, when, where, why and how. As scholars address these key questions, we believe that organizational resilience scholarship may advance and continue to mature.

We recognize that this volume does not cover the universe of resilience scholarship and practice, yet our hope is that this Research Handbook will advance our collective conversation about the meaning, foundations, dimensions, characteristics, attributes and definitions of resilience. We believe the work represented herein will foster greater understanding about how to conceptualize and study resilience, thus providing needed clarification and rigor to resilience scholarship.

\section{REFERENCES}

Gittell, J.H., Cameron, K., Lim, S. and Rivas, V. (2006). Relationships, layoffs, and organizational resilience: Airline industry responses to September 11. The Journal of Applied Behavioral Science, 42(3), 300-329.

Guarnieri, F. and Travadel, S. (2018). Un récit de Fukushima. Paris: Presses Universitaires de France.

Janoff-Bulman, R. (1985). The aftermath of victimization: Rebuilding shattered assumptions. In C.R. Figley (ed.), Trauma and its wake: The study and treatment of posttraumatic stress disorder (pp. 15-35). New York: Brunner/Mazel.

Janoff-Bulman, R. (1992). Shattered assumptions: Towards a new psychology of trauma. New York: Free Press.

Maitlis, S. (2012). Posttraumatic growth: A missed opportunity for positive organizational scholarship. In K.S. Cameron and G. Spreitzer (eds), The Oxford handbook of positive organizational scholarship. Oxford: Oxford University Press.

Powley, E.H. (2009). Reclaiming resilience and safety: Resilience activation in the critical period of crisis. Human Relations, 62(9), 1289-326.

Sutcliffe, K.M. and Vogus, T.J. (2003). Organizing for resilience. In K.S. Cameron, J.E. Dutton and R.E. Quinn (eds), Positive organizational scholarship: Foundations of a new discipline (pp. 94-110). San Francisco, CA: Berrett-Koehler.

Tugade, M.M. and Fredrickson, B.L. (2004). Resilient individuals use positive emotions to bounce back from negative emotional experiences. Journal of Personality and Social Psychology, 86(2), 320-33.

Westphal, M. and Bonanno, G.A. (2007). Posttraumatic growth and resilience to trauma: Different sides of the same coin or different coins? Applied Psychology, 56(3), 417-27.

Zolli, A. and Healy, A.M. (2012). Resilience: Why things bounce back. New York: Free Press. 